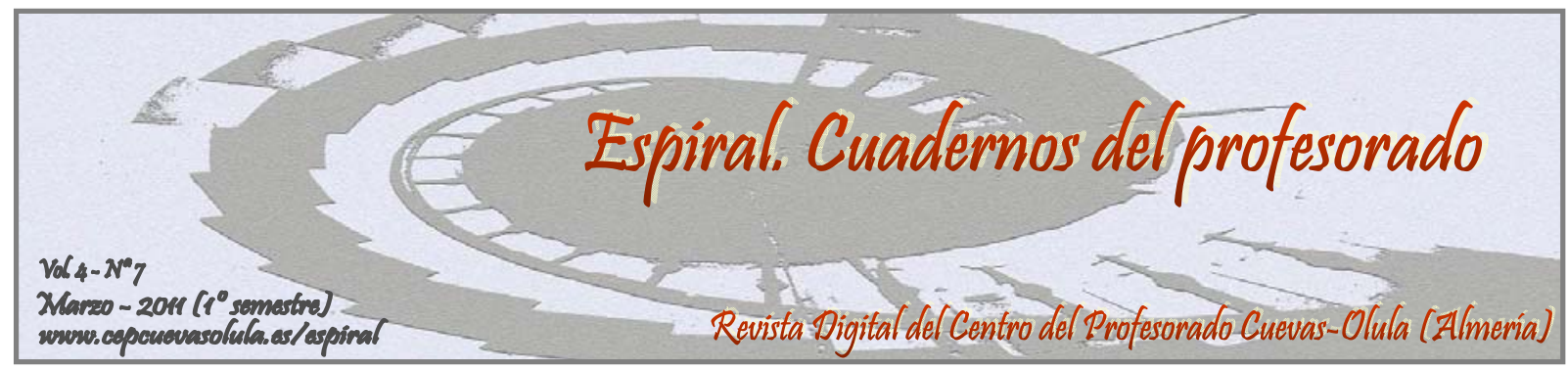

\title{
MEDIDAS EDUCATIVAS Y ORGANIZATIVAS DE ATENCIÓN A LA DIVERSIDAD QUE MEJORAN EL RENDIMIENTO ESCOLAR EN EL IES SIERRA DE LOS FILABRES DE SERÓN (ALMERÍA)
}

\author{
EDUCATIONAL AND ORGANIZATIONAL MEASURES ADDRESSING \\ DIFFERENTIATED INSTRUCTION THAT IMPROVE ACADEMIC \\ PERFORMANCE IN THE IES SIERRA DE LOS FILABRES, IN SERÓN (ALMERIA)
}

Manuel Navarro Reyes

IES Jiménez Montoya de Baza, Granada, España

RESUMEN: Este artículo pretende demostrar cómo a través de la adopción de medidas educativas y organizativas adecuadas, seleccionadas y bien planificadas, se puede influir positivamente sobre el rendimiento escolar del alumnado en la Enseñanza Secundaria Obligatoria. La experiencia ha sido realizada en el IES Sierra de los Filabres de la localidad almeriense de Serón, durante los cursos académicos 2008-2009 y 2009-2010. El punto de partida fueron los datos obtenidos del Sistema de Gestión de Centros (Séneca), para el curso escolar 2007-2008, sobre promoción, titulación y rendimiento escolar en las materias instrumentales de lengua, matemáticas e inglés. El equipo directivo del centro decidió, al amparo de la nueva ley aprobada sobre atención a la diversidad (Orden de 25 de Julio de 2008), aplicar una serie de medidas organizativas con intención de estudiar su influencia sobre la mejora en el rendimiento escolar del alumnado, para lo cual se estableció un horizonte de dos cursos escolares. El siguiente trabajo, por tanto, recoge cómo se realizó la experiencia y los resultados que se obtuvieron.

Palabras clave: rendimiento escolar, atención a la diversidad, medidas organizativas, promoción, titulación, materias instrumentales.

ABSTRACT: This article seeks to show how we can positively influence secondary students' academic performance by adopting appropriate and well-planned educational and organizational measures. This study has been carried out at the IES Sierra de los Filabres, in Serón (Almeria), during 2008-2009 and 2009-2010. The project was initially inspired by the results obtained from the School Management System (Séneca), for 2007-2008, with respect to promotion, qualifications and level of performance in the core subjects of Spanish, Maths and English. The school management team decided, under the new law about differentiated instruction (25th July, 2008), to implement a series of organizational measures in order to study their influence on the improvement of students' academic performance; the intervention period set was two years. The following study, therefore, describes how the project was carried out and the results that were obtained.

Key words: academic performance differentiated learning, organizational measures, promotion, qualifications, core subjects. 
Navarro Reyes, M. (2011). Medidas educativas y organizativas de atención a la diversidad que mejoran el rendimiento escolar en el IES Sierra de los Filabres de Serón (Almería). Espiral. Cuadernos del Profesorado [en línea], 4(7), 29-41. Disponible en: $\mathrm{http}: / / \mathrm{www} . c e p c u e v a s o l u l a . e s /$ espiral.

Fecha de recepción: 12/08/2010

Fecha de aceptación: 17/11/2010
Enviar correspondencia a: manare66@hotmail.com

\section{1.- INTRODUCCIÓN.}

La educación, y por tanto, el sistema educativo, es responsable de integrar y normalizar la formación y la vida de todas las personas sean cual sean sus diferencias. La calidad que puede ofrecer un sistema educativo no se mide únicamente por los logros alcanzados por aquellos alumnos/as bien dotados, sino, sobre todo, por la capacidad que tiene dicho sistema a la hora de ofrecer, proponer y aplicar un diseño y una práctica educativa capaz de dar respuesta a la diversidad de capacidades, intereses y motivaciones de todos ellos y ellas. "No son los alumnos/as con dificultades quienes tienen que adaptarse o "conformarse" a lo que pueda enseñarles una enseñanza general, planificada y desarrollada para satisfacer las necesidades educativas habituales de la mayoría del alumnado, sino que es la enseñanza la que debe adecuarse al modo y manera que permite a cada alumno particular progresar en función de sus capacidades y con arreglo a que sus necesidades sean especiales o no" (Arnaiz, 1997).

Todos los alumnos/as no aprenden con la misma facilidad, ni al mismo ritmo, ni presentan la misma motivación. Si estas diferencias se encuentran en los alumnos/as individualmente, cuánto más en un aula o en el propio centro. Se trata entonces de encontrar la forma de ofrecer un currículo común para todos/as pero diversificado. Es decir, ofrecer a cada uno/a lo que verdaderamente necesita para su propio crecimiento personal, social y educativo.

La aparición de la LOGSE, (1990), (Ley orgánica de ordenación general del sistema educativo en España), introdujo a nivel formal un cambio en esta perspectiva y planteó una visión curricular en la respuesta individualizada a las necesidades educativas especiales de los alumnos/as. Después de algunos años de aplicación y a pesar del impulso que supuso, encontró en la práctica un reto difícil y en algunas ocasiones contradictorio con algunos valores sociales todavía vigentes.
La actual ley de educación LOE, (2007), (Ley orgánica de educación), volvió a profundizar en la necesidad de atender a la diversidad como factor de calidad del sistema educativo. El necesario ajuste de la oferta pedagógica a las capacidades, intereses $\mathrm{y}$ necesidades del alumnado, se postula como eje vertebrador de esta nueva concepción educativa. Ante nosotros aparecen herramientas adecuadas para paliar posibles desigualdades en las condiciones de acceso a la educación. Se trata, por tanto, de aunar esfuerzos en la búsqueda de aquellas medidas que realmente contribuyan a ello.

Son pocos los trabajos que han relacionado la utilización de las medidas de organización (desde el punto de vista de atención a la diversidad) con las mejoras de los rendimientos de los alumnos/as.

Un estudio descriptivo-diferencial, realizado por profesorado de la Universidad de Sevilla (Víctor Álvarez y otros. 2002), intentó conocer las actuaciones que llevaban a cabo los centros en relación a la atención a la diversidad, los destinatarios de éstas y la opinión del profesorado en relación a las dimensiones organizativas e institucionales.

Las conclusiones fueron que la adaptación curricular y el refuerzo educativo eran las medidas más frecuentemente utilizadas y que la individualización de la enseñanza y la adecuación de los objetivos eran en las que más participaban. Los docentes, también destacaban como medidas organizativas las relacionadas con la acción tutorial y la estabilidad del profesorado.

Otro estudio realizado en el IES Juan Antonio Castro, de Talavera de la Reina (Toledo), abordó la utilización de los "Agrupamientos flexibles" como medida organizativa a aplicar en la mejora de los rendimientos (Departamento de Orientación y Equipo Directivo. 2010). Los grupos sometidos a esta medida demostraron una mejoría del $20 \%$ 
de aprobados en las asignaturas instrumentales frente a los que no se le aplicaron.

En ninguno de los dos casos se abarcó dicha relación desde el punto de vista de la organización curricular, no se adoptaron medidas previas que afectaran a la matriculación del alumnado de nueva incorporación y por tanto no se daba respuesta a las necesidades educativas desde una dimensión global.

En el año 2008, la Consejería de Educación de la Junta de Andalucía, aprobó la Orden de 25 de Julio de Atención a la Diversidad. El equipo directivo y claustro de profesores del IES Sierra de los Filabres de Serón, asumimos el reto de poner en práctica la multitud de posibilidades de las que dotaba la ley, especialmente las relacionadas con la organización del currículum y las consecuencias, en cuanto al rendimiento escolar, que estas actuaciones supondrían. El primer paso consistió en una valoración inicial del rendimiento del alumnado, posteriormente se eligieron las opciones más válidas, organizando protocolos de matriculación para el alumnado de primero y segundo de la ESO El periodo de aplicación y evaluación se estableció en dos años, coincidiendo con la misma fase de desarrollo del "Plan de Calidad" (programa de calidad y mejora de los rendimientos escolares en los centros docentes públicos), aunque se estableció una revisión profunda tras la evaluación del primer curso escolar.

En las siguientes líneas desarrollamos cómo se realizó la experiencia y las conclusiones más relevantes que se obtuvieron.

\section{2.- PRESENTACIÓN DEL PROYECTO.}

\section{1.- Contextualización. Características del alumnado del centro}

La edad de los alumnos/as del IES de Serón está comprendida entre los 12 y los 16 años. El 57\% de los alumnos de este instituto son varones, mientras que el $47 \%$ son mujeres. El nivel sociológico general de los alumnos/as de este IES es medio-bajo. El grado de absentismo es muy bajo, por lo que no se considera un factor significativo. El abandono escolar no existe en alumnos menores de 16 años, a partir de aquí si lo es y aunque las cifras son muy bajas y no demasiadas significativas, es necesario hacer hincapié en la motivación y orientación de los alumnos como medida para disminuir este aspecto.
En cuanto a la convivencia destacar que casi no se producen incidentes y sobre todo no son graves. Los datos sacados del programa de gestión Séneca y los obtenidos del observatorio para la convivencia realizado por la Agencia Andaluza de Evaluación Educativa (Junta de Andalucía, 2010), nos indican que el $88 \%$ de nuestro alumnado no ha cometido nunca conductas contrarias a la convivencia y que el porcentaje de los que si las cometen (12\%) es inferior en un tercio al que se produce de media en la Comunidad Autónoma.

El índice de matriculación en estudios postobligatorios después de acabar la ESO se sitúa en el $80 \%$.

Aunque el centro se encuentra enclavado en el casco urbano de Serón, concretamente a la entrada del pueblo dirección norte, existe un grupo de alumnos (27-09/10) que viven en barriadas apartadas de esta localidad, siendo la más lejana la situada a unos 20 kilómetros. Necesitando todos ellos de transporte escolar.

En cuanto a la diversidad social, empiezan a existir alumnos/as inmigrantes y en estos últimos años ya suponen un $20 \%$ del alumnado total.

La participación de los alumnos/as en las actividades escolares y extraescolares no es muy alta, debido a la poca cultura participativa que existe, al igual que ocurre con las familias, siendo, por lo tanto, el tema de la participación un elemento relevante y a tener en cuenta en los objetivos particulares del centro.

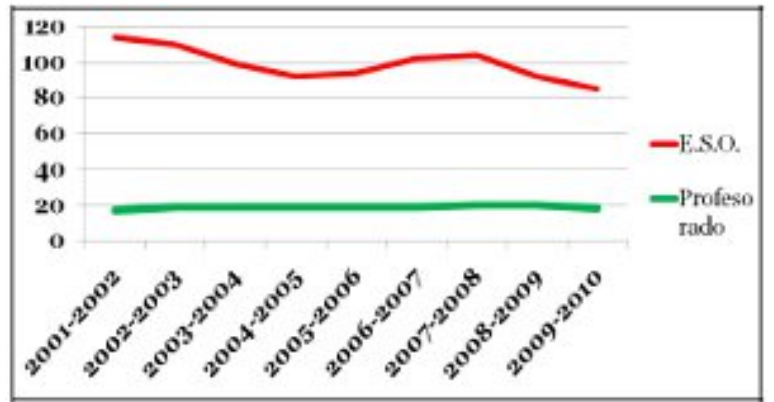

Figura 1.- Ratio (relación alumnado/profesorado; expresado en número absolutos).

Por tanto, el alumnado responde a las características propias de la dispersión geográfica $\mathrm{y}$ de la procedencia de un entorno eminentemente rural (nivel socio-cultural medio-bajo, escasez de estímulos sociales que valoren los aprendizajes intelectuales, pocas posibilidades de contactar con grandes centros culturales -muy retirados de la comarca-, etc.). Como dato positivo resaltar que con la bajada de la matriculación y el 
mantenimiento del número de profesores, la relación alumno/a-profesor/a es excelente situándose en $5 \mathrm{y}$ habiendo evolucionado en los últimos 9 años en 1.5 (figura 1), hecho que ha permitido mejorar las posibilidades de individualización de los procesos de enseñanza.

En cuanto a la convivencia destacar que casi no se producen incidentes y sobre todo no son graves. Los datos sacados del programa de gestión Séneca y los obtenidos del observatorio para la convivencia realizado por la Agencia Andaluza de Evaluación Educativa (Junta de Andalucía, 2010), nos indican que el $88 \%$ de nuestro alumnado no ha cometido nunca conductas contrarias a la convivencia y que el porcentaje de los que si las cometen $(12 \%)$ es inferior en un tercio al que se produce de media en la Comunidad Autónoma.

El índice de matriculación en estudios postobligatorios después de acabar la ESO se sitúa en el $80 \%$.

\section{2.- Origen de la experiencia. Valoración inicial.}

El punto de partida de la experiencia fue la realización de una valoración inicial al alumnado del centro. Nos sirvieron de referencia los datos del rendimiento de éstos, extraídos del sistema de gestión "Séneca", así como los resultados de las pruebas de diagnóstico, todas ellas del curso escolar 2007-2008. El estudio se centró fundamentalmente sobre los dos primeros cursos de la E.S.O., y sobre las materias instrumentales (lengua castellana, matemáticas e inglés).

Tabla 1. Comparativa alumnado promocionado del centro y alumnado andaluz, año 2007.

\begin{tabular}{lccc}
\hline & \multicolumn{2}{c}{$\begin{array}{c}\text { SERÓN } \\
\mathbf{2 0 0 7 - 2 0 0 8}\end{array}$} & $\begin{array}{c}\text { ANDALUCÍA } \\
\text { (Junta de Andalucía, } \\
\text { 2009) }\end{array}$ \\
\hline $\mathbf{1}^{\mathbf{0}}$ ESO & $56.3 \%$ & $\downarrow$ & $78.5 \%$ \\
$\mathbf{2}^{\mathbf{0}}$ ESO & $76.2 \%$ & $\uparrow$ & $75.6 \%$ \\
$\mathbf{3}^{\mathbf{0}}$ ESO & $68.0 \%$ & $\downarrow$ & $72.0 \%$ \\
$\mathbf{4}^{\mathbf{0}}$ ESO & $90.9 \%$ & $\uparrow$ & $76.8 \%$ \\
TOTAL & $72.8 \%$ & $\downarrow$ & $75.7 \%$ \\
\hline
\end{tabular}

El índice de alumnado que promocionó o tituló de forma global en el centro durante este curso fue del $72.83 \%$, cifra que se sitúa $2.80 \%$ por debajo de la media andaluza que en el 2007 era de un $75.72 \%$ (tabla 1 ). Su distribución por cursos (figura 2) nos decía que los peores resultados se daban en $1^{\circ}$ de E.S.O. con tan sólo un $56.25 \%$ de éxito escolar y en $3^{\circ}$ de E.S.O. con un $68 \%$.

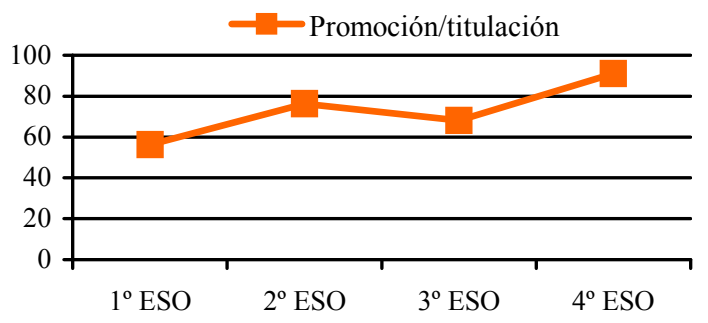

Figura 2.- Alumnado promocionado por curso en 2007-2008.

En lengua castellana el porcentaje de alumnos/as que no superaba la materia era de $31.25 \%$, mientras que en segundo se elevaba al $46.6 \%$.

En matemáticas la cifra en primero estaba en el $37.5 \%$ y también se elevaba en el segundo curso hasta alcanzar el $40 \%$. En inglés el fracaso era ligeramente menor situándose en el $38.1 \%$ para primero y en $34 \%$ para segundo (tabla 2 ).

Tabla 2.- Resultados asignaturas instrumentales en el curso 2007-2008.

\section{CURSO 2007-2008}

\begin{tabular}{cccc}
\hline & $\begin{array}{c}\text { Suspensos } \\
\text { en Lengua }\end{array}$ & $\begin{array}{c}\text { Suspensos en } \\
\text { matemáticas }\end{array}$ & $\begin{array}{c}\text { Suspensos en } \\
\text { inglés }\end{array}$ \\
\cline { 2 - 4 } $\mathbf{1}^{\circ} \mathbf{E S O}$ & $31.2 \%$ & $37.5 \%$ & $38.1 \%$ \\
$\mathbf{2}^{\circ} \mathbf{E S O}$ & $46.6 \%$ & $40.0 \%$ & $40.0 \%$ \\
TOTAL & $38.6 \%$ & $38.7 \%$ & $34.0 \%$ \\
\hline
\end{tabular}

Por otra parte la evaluación de diagnóstico del curso escolar en cuestión (Junta de Andalucía, 2009) nos daba unos resultados buenos en lengua castellana, por encima de la media Andaluza, mientras en matemáticas los situaba ligeramente por debajo. Estos resultados coincidían con los de rendimiento académico en esta última asignatura, sin embargo en lengua castellana no parecía así (figura 3).

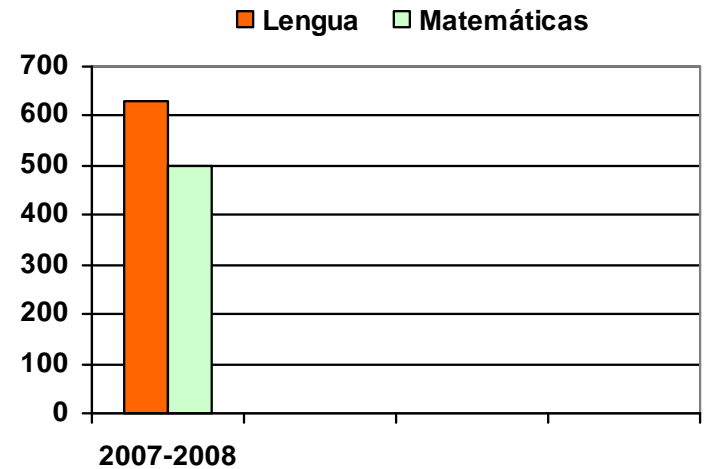

Figura 3.- Puntuación transforma en las Pruebas de Diagnóstico. Curso 2007-2008 


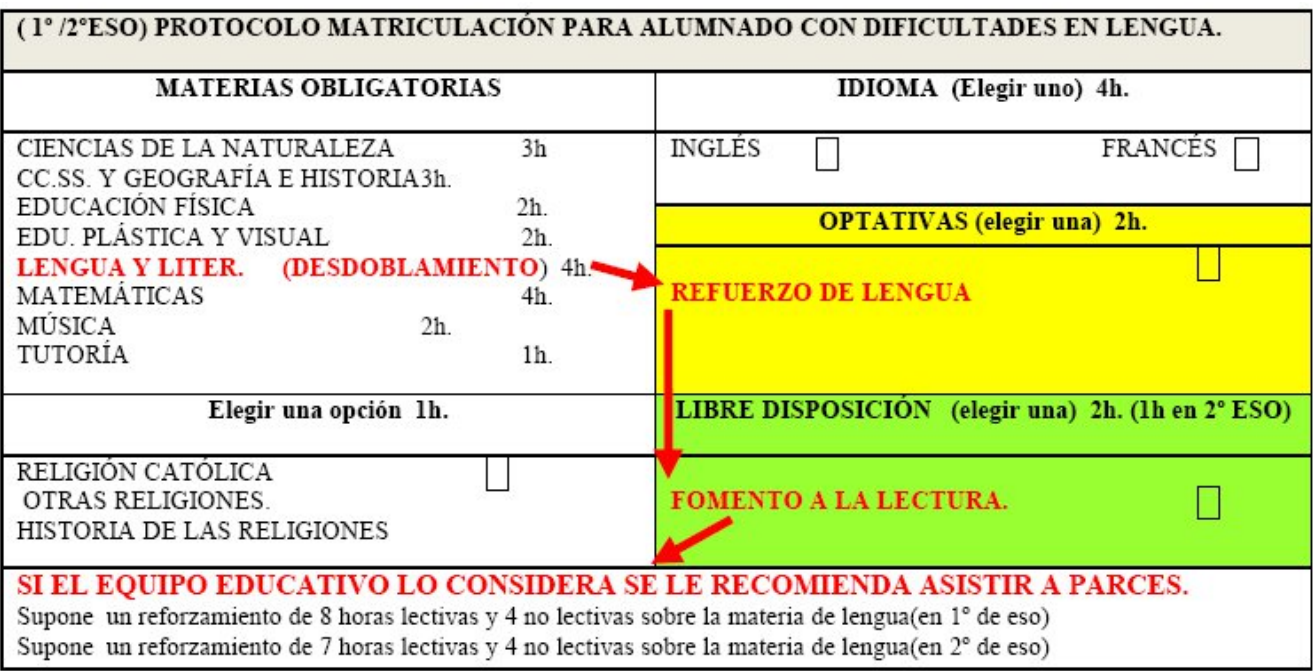

Figura 4.- Protocolo de matriculación para alumnado con dificultades en Lengua Castellana

\section{3.- Objetivos.}

Después de la valoración inicial establecimos los siguientes objetivos:

$1^{\circ}$.- Tratar de elevar la tasa de alumnado que promocionaba anualmente de forma global. Para el 2007-08 situada en el $72.83 \%$. Cifra ya de por sí cercana a la media andaluza, de manera que nuestras expectativas eran el elevarla en un porcentaje que rondaba entre el 2 y el $5 \%$.

$2^{\circ}$.- Elevar la tasa de alumnado que promocionaba específicamente en $1^{\circ}$ de E.S.O., para el curso 2007-08 situada en el 56.25\%.

$3^{\circ}$.-Elevar el éxito escolar en la asignatura de lengua castellana y específicamente en segundo curso donde el fracaso se situaba en el $46.6 \%$.

$4^{\mathrm{o}}$.-Elevar el éxito escolar en la asignatura de matemáticas en ambos cursos.

$5^{\circ}$.- Elevar el éxito escolar en la asignatura de inglés en ambos cursos.

$6^{\circ}$.- Facilitar la adaptación del alumnado al entorno escolar y al aula.

$7^{\circ}$.- Informar a las familias ante el proceso de matriculación de sus hijos/as.

\section{3.- DESARROLLO.}

3.1.- Actuaciones desarrolladas. Curso 20082009.

Se tomaron las medias de carácter organizativo siguientes:
1.- Desdoblamiento de la asignatura de lengua castellana en $1^{\circ}$ de E.S.O. (aproximadamente grupos de 15 alumnos/as).

2.- Desdoblamiento de la asignatura de matemáticas en $1^{\circ}$ de E.S.O. (aproximadamente grupos de 15 alumnos/as).

3.- Triple desdoblamiento de la asignatura de lengua castellana en $2^{\circ}$ de E.S.O. (aproximadamente grupos de 10 alumnos/as).

4.- Desdoblamiento de la asignatura de matemáticas en $2^{\circ}$ de E.S.O. (aproximadamente grupos de 15 alumnos/as).

5.- Creación de un programa de refuerzo en la asignatura de lengua para $1^{\circ}$ y $2^{\circ}$ de E.S.O.

6.- Creación de un programa de refuerzo en la asignatura de matemáticas para $1^{\circ}$ y $2^{\circ}$ de E.S.O.

7.- Creación de la asignatura de libre disposición "Fomento a la Lectura".

8.- Aplicación del programa P.A.R.C.E.S. (*) (Programa de apoyo y refuerzo para secundaria).

Se tomaron las medias de carácter individual siguientes:

1.- Programas de refuerzo de las materias pendientes de otros cursos (se designó la figura de un tutor de pendientes para la E.S.O.).

2.- Planes específicos para el alumnado que no promocionó.

3.- Programa de adaptación curricular y apoyos. 
Medidas educativas y organizativas de atención a la diversidad que mejoran el rendimiento escolar en el IES Sierra de los Filabres de Serón (Almería)

\begin{tabular}{|c|c|}
\hline \multicolumn{2}{|c|}{$\left(1^{\circ} / 2^{\circ} \mathrm{ESO}\right)$ PROTOCOLO MATRICULACIÓN PARA ALUMNADO CON DIFICULTADES EN MATEMÁTICAS } \\
\hline MATERIAS OBLIGATORIAS & IDIOMA (Elegir uno) 4h. \\
\hline \multirow{3}{*}{ 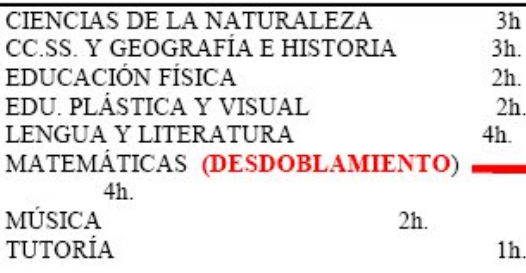 } & FRANCÉS $\square$ \\
\hline & OPTATIVAS (elegir una) $2 \mathrm{~h}$. \\
\hline & REFUERZO DE MATEMÁTICAS \\
\hline Elegir una opción lh. & LIBRE DISPOSICIÓN (elegir una) $2 \mathrm{~h}$. (lh en $2^{\circ}$ ESO) \\
\hline $\begin{array}{l}\text { RELIGIÓN CATÓLICA } \\
\text { OTRAS RELIGIONES. } \\
\text { HISTORIA DE LAS RELIGIONES }\end{array}$ & ESTRATEGIAS MATEMÁTICAS \\
\hline \multicolumn{2}{|c|}{$\begin{array}{l}\text { SI EL EQUIPO EDUCATIVO LO CONSIDERA SE LE RECOMIENDA ASISTIR A PARCES } \\
\text { Supone un reforzamiento de } 8 \text { horas lectivas y } 4 \text { no lectivas sobre la materia de matemáticas }\left(1^{\circ} \text { de eso). }\right. \\
\text { Supone un reforzamiento de } 7 \text { horas lectivas y } 4 \text { no lectivas sobre la materia de matemáticas }\left(2^{\circ} \text { de eso). }\right.\end{array}$} \\
\hline
\end{tabular}

Figura 5.- Protocolo de matriculación para alumnado con dificultades en Matemáticas.

\section{2.- Metodología.}

$1^{\circ}$.- Reunión informativa con las familias del alumnado de primero y segundo de ESO para explicar cómo matricularse de forma correcta atendiendo a la nueva normativa. Explicación de todas las posibilidades, optativas, itinerarios, ofrecidos por nuestro centro.

$2^{\circ}$.- Estudio del informe de tránsito para cotejarlo con las matrículas realizadas.

$3^{\text {o.- }}$ Establecimiento de protocolos de matriculación.

\section{3.- Protocolos de matriculación.}

\section{A.-Alumnado con dificultades en lengua castellana (figura 4):}

$1^{\circ}$ Matriculado en grupo de desdoblamiento.

$2^{\circ}$ Matriculado en refuerzo de lengua.

$3^{\circ}$ Matriculado en Fomento a la Lectura.

$4^{\circ}$ Opcionalmente y si era propuesto por el equipo educativo, se incluía en el programa P.A.R.C.E.S. (programa de apoyo y refuerzo educativo para secundaria).

B-Alumnado con dificultades en matemáticas (figura 5):

$1^{\circ}$ Matriculado en grupo de desdoblamiento.

$2^{\circ}$ Matriculado en refuerzo de matemáticas.

$3^{\circ}$ Matriculado en estrategias matemáticas.

$4^{\circ}$ Opcionalmente y si era propuesto por el equipo educativo, se incluía en el programa P.A.R.C.E.S. (programa de apoyo y refuerzo

\begin{tabular}{|c|c|}
\hline \multicolumn{2}{|c|}{$\begin{array}{l}\text { ( } 1^{\circ} \text { ESO) PROTOCOLO MATRICULACIÓN PARA ALUMNADO CON DIFICULTADES EN MATEMÁTICAS Y } \\
\text { LENGUA CASTELLANA. }\end{array}$} \\
\hline MATERIAS OBLIGATORIAS & IDIOMA (Elegir uno) $4 \mathrm{~h}$. \\
\hline \multirow{3}{*}{ 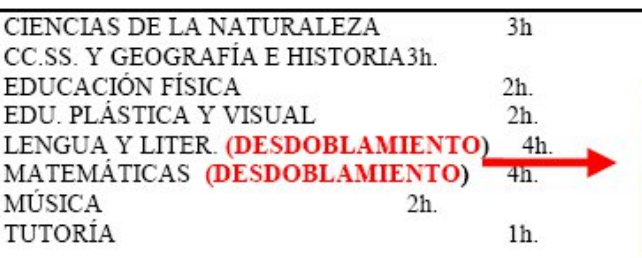 } & $\begin{array}{ll}\text { INGLÉS } & \text { FRANCÉS } \square \\
& \end{array}$ \\
\hline & OPTATIVAS (elegir una) $2 \mathrm{~h}$. \\
\hline & REFUERZO DE LENGUA \\
\hline Elegir una opción lh. & LIBRE DISPOSICIÓN (elegir una) $2 \mathrm{~h}$. (lh en $2^{\circ}$ ESO) \\
\hline $\begin{array}{l}\text { RELIGIÓN CATÓLICA } \\
\text { OTRAS RELIGIONES. } \\
\text { HISTORIA DE LAS RELIGIONES }\end{array}$ & ESTRATEGIAS MATEMÁTICAS \\
\hline $\begin{array}{l}\text { SI EL EQUIPO EDUCATIVO LO CONSIDERA } \\
\text { Supone un reforzamiento de } 8 \text { horas lectivas y } 4 \text { no } 1 \\
\text { Supone un reforzamiento de } 7 \text { horas lectivas y } 4 \text { no } 1\end{array}$ & $\begin{array}{l}\text { OMIENDA ASISTIR A PARCES } \\
\text { la materia de lengua y matemáticas }\left(1^{\circ} \text { de ESO) }\right. \\
\text { e la materia de lengua y matemáticas }\left(2^{\circ} \text { de ESO). }\right.\end{array}$ \\
\hline
\end{tabular}

Figura 6.- Protocolo de matriculación para alumnado con dificultades en Matemáticas y Lengua Castellana. 
educativo para secundaria).

C.-Alumnado con dificultades en lengua $y$ matemáticas (figura 6):

$1^{\circ}$ Matriculado en grupo de desdoblamiento de lengua y matemáticas.

$2^{\circ}$ Matriculado en refuerzo de lengua.

$3^{\circ}$ Matriculado en Estrategias matemáticas.

$4^{\circ}$ Opcionalmente y si era propuesto por el equipo educativo, se incluía en el programa P.A.R.C.E.S. (programa de apoyo y refuerzo educativo para secundaria.

Tabla 2.- Comparativa resultados alumnado promocionado en los cursos 2007-2008 y 2008-2009.

\begin{tabular}{lccccc}
\hline & \multicolumn{2}{c}{$\begin{array}{c}\text { SERÓN } \\
\mathbf{2 0 0 7 - 2 0 0 8}\end{array}$} & \multicolumn{2}{c}{$\begin{array}{c}\text { SERÓN } \\
\mathbf{2 0 0 7 - 2 0 0 8}\end{array}$} & $\begin{array}{c}\text { ANDALUCÍA } \\
\text { (Junta de } \\
\text { Andalucía, 2009) }\end{array}$ \\
\hline $\mathbf{1}^{\mathbf{0}}$ ESO & $56.6 \%$ & $\downarrow$ & $74.0 \%$ & $\uparrow$ & $78.5 \%$ \\
$\mathbf{2}^{\mathbf{o}}$ ESO & $76.2 \%$ & $\uparrow$ & $57.0 \%$ & $\downarrow$ & $75.6 \%$ \\
$\mathbf{3}^{\mathbf{0}}$ ESO & $68.0 \%$ & $\downarrow$ & $89.0 \%$ & $\uparrow$ & $72.0 \%$ \\
$\mathbf{4}^{\mathbf{o}}$ ESO & $90.9 \%$ & $\uparrow$ & $100.0 \%$ & $\uparrow$ & $76.8 \%$ \\
TOTAL & $72.8 \%$ & $\downarrow$ & $80.0 \%$ & $\uparrow$ & $75.7 \%$ \\
\hline
\end{tabular}

\section{D.-Alumnado con dificultades en inglés:}

Sólo se aplicaron las adaptaciones curriculares pertinentes así como los programas de recuperación de aprendizajes no adquiridos. No se pudo realizar ninguna actuación más de carácter organizativo por falta de profesorado.
Se consiguió el objetivo de elevar la tasa total de alumnado que promocionaba o titulaba $(80 \%)$, mucho más de lo programado (entre 2.80 y $5 \%$ ) y un $5 \%$ por encima de la media andaluza $(75.72 \%)$ (tabla 2).) Otro de los objetivos sobradamente cumplido fue la mejora de alumnado con éxito escolar en $1^{\circ}$ de ESO, elevando la cifra del $56.25 \%$ al $76.2 \%$. Es posible que estas cifras tan altas se vieran influenciadas por el alto grado de mejora detectado en tercero y cuarto curso que indiscutiblemente contribuyó de forma notoria a la elevación de los porcentajes.

El éxito en la asignatura de lengua castellana, específicamente en segundo curso también se alcanzó, de unos valores de fracaso entorno al $46.6 \%$ se redujo a sólo el $29.6 \%$. De un porcentaje global entre $1^{\circ}$ y $2^{\circ}$ curso del $38.6 \%$ se consiguió reducir al valor de $23.7 \%$.

En la asignatura de matemáticas no parece haber cambios significativos, a pesar de bajar la tasa de fracaso en $1^{\circ}$ de ESO, ya que en segundo se ve incrementada dicha tasa casi en un $10 \%$.

Para la asignatura de inglés y dada la carencia de actuaciones para su mejora, los

Tabla 3.- Comparación de los resultados en las instrumentales de los dos cursos escolares.

\begin{tabular}{ccccccc}
\hline & $\begin{array}{c}\text { Suspensos } \\
\text { en Lengua } \\
(\mathbf{2 0 0 7 - 2 0 0 8 )}\end{array}$ & $\begin{array}{c}\text { Suspensos } \\
\text { en Lengua } \\
(\mathbf{2 0 0 8 - 2 0 0 9 )}\end{array}$ & $\begin{array}{c}\text { Suspensos } \\
\text { en matemáticas } \\
(\mathbf{2 0 0 7 - 2 0 0 8 )}\end{array}$ & $\begin{array}{c}\text { Suspensos } \\
\text { en matemáticas } \\
(\mathbf{2 0 0 8 - 2 0 0 9 )}\end{array}$ & $\begin{array}{c}\text { Suspensos } \\
\text { en inglés } \\
(\mathbf{2 0 0 7 - 2 0 0 8 )}\end{array}$ & $\begin{array}{c}\text { Suspensos } \\
\text { en inglés } \\
(\mathbf{2 0 0 8}-2009)\end{array}$ \\
\hline $\mathbf{1}^{\mathbf{0}}$ ESO & $31.25 \%$ & $17.8 \%$ & $37.5 \%$ & $28.5 \%$ & $38.1 \%$ & $25.0 \%$ \\
$\mathbf{2}^{\mathbf{0}}$ ESO & $46.6 \%$ & $29.6 \%$ & $40.0 \%$ & $51.8 \%$ & $40.0 \%$ & $51.8 \%$ \\
TOTAL & $38.6 \%$ & $23.7 \%$ & $38.7 \%$ & $40.15 \%$ & $34.05 \%$ & $38.4 \%$ \\
\hline
\end{tabular}

(*) El programa P.A.R.C.E.S. (programa de apoyo y refuerzo educativo) se impartió durante todo el curso escolar. Consistió en clases de refuerzo de las asignaturas instrumentales-lengua y matemáticas- en horario de tarde y con grupos pequeños-máximo 6 alumnos/as.

Las clases fueron impartidas por profesorado con las licenciaturas de filología hispánica y matemáticas, no siendo personal del centro.

El alumnado que se favoreció de este programa estuvo propuesto por el equipo educativo correspondiente, teniendo los padres/madres que autorizar dicha inclusión.

\section{4.- Conclusiones al término del primer curso escolar (2008-2009).}

resultados se mantuvieron de un curso a otro, el $38.4 \%$ de fracaso, un $4 \%$ más que el año anterior (tabla 3).

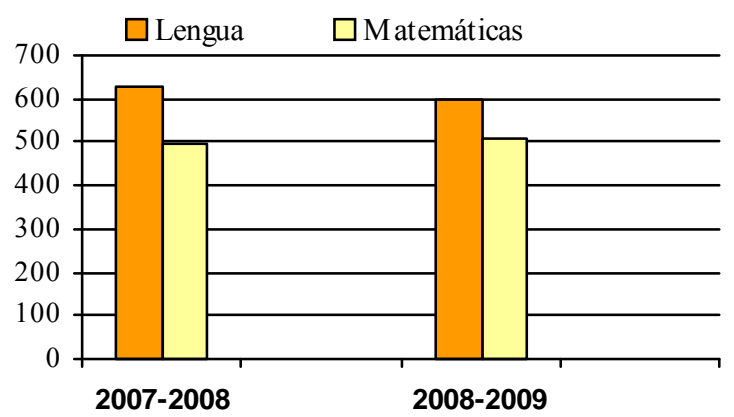

Figura 7.- Resultados pruebas de diagnóstico cursos 2007-2008 y 2008-2009. 
En cuanto a la evaluación de diagnóstico se refiere (figura 7) (Junta de Andalucía, 2009), los resultados en lengua castellana se mantuvieron por encima de los 600 puntos transformados mientras en matemáticas siguieron estando en la media de los 500 puntos. Las pruebas en realidad se realizaron a alumnos/as del tercer curso escolar sobre los que no se había actuado (el proyecto abarcaba $1^{\circ}$ y $2^{\circ}$ de ESO).

La convivencia siguió manteniéndose dentro de una tónica buena.

En resumen, se mejoraron los resultados en la asignatura de lengua castellana, tanto en primero como en segundo curso. Se mejoraron las tasas de promoción-titulación en el centro así como específicamente las del primer curso de la E.S.O.

Los resultados de las pruebas de diagnóstico no se vieron modificados con respecto al año anterior de forma significativa, la convivencia mejoró en tanto en cuanto se produjeron menos incidentes.

Se mejoró el rendimiento de los alumnos/as de primero en las asignaturas de matemáticas e inglés pero no globalmente ya que en el segundo curso empeoraron. No parecieron haber producido las medidas aplicadas modificaciones de los resultados en estas asignaturas.

\begin{tabular}{|l|l|l|l|l|l|l|l|l|l|}
\hline $\begin{array}{l}\mathrm{N}^{\circ} \\
\text { alumnos/as }\end{array}$ & \multicolumn{2}{|l|}{$\begin{array}{l}\text { Desdoblamiento } \\
\text { lengua }\end{array}$} & \multicolumn{2}{l}{$\begin{array}{l}\text { Desdoblamiento } \\
\text { matemáticas }\end{array}$} & $\begin{array}{l}\text { Desdoblamiento } \\
\text { C. naturales }\end{array}$ & \multicolumn{2}{|l|}{$\begin{array}{l}\text { Agrupamiento } \\
\text { flexible inglés }\end{array}$} \\
\hline $1^{\circ}$ & & & 11 & 11 & 11 & 11 & 10 & 12 \\
\hline $2^{\circ}$ & 15 & 14 & 10 & 10 & 9 & 14 & 15 & 16 & 13 \\
\hline
\end{tabular}

Figura 8.- Distribución del alumnado en los desdoblamientos.

El E.T.C.P. reunido al final de este curso escolar emitió unas conclusiones en la memoria del Plan de atención a la diversidad, que sirvieron de base para la planificación del siguiente año. Estas fueron las siguientes:

1.-La bajada de la ratio a través de desdoblamientos en $1^{\circ}$ y $2^{\circ}$ de E.S.O. en asignaturas instrumentales mejora los resultados alcanzados.

2.-Búsqueda y aplicación de otras medidas de atención a la diversidad en $1^{\circ}$ y $2^{\circ}$, fundamentalmente en matemáticas.

3.-Aumentar el número de horas de lectura del alumnado.

4.-Potenciar los idiomas en lengua extranjera, tanto a través de desdoblamientos, refuerzos, así como en asignaturas de libre disposición.

\section{5.- Actuaciones desarrolladas. Curso escolar 2009-2010.}

Se decidieron mantener todos los objetivos generales del programa, modificando sólo las actuaciones a seguir. El documento de partida fueron las conclusiones realizadas por el equipo técnico de coordinación pedagógica al término del curso escolar.

Se tomaron las medias de carácter organizativo siguientes:

1.-Petición de cambio de un profesor/a de lengua castellana por uno/a de inglés.

2.-Continuación de forma generalizada de los desdoblamientos en $1^{\circ}$ y $2^{\circ}$ en las materias de lengua y matemáticas. En $2^{\circ}$ se hace un triple desdoblamiento en matemáticas. En los dos cursos se realiza también en la asignatura de ciencias naturales (figura 8).

3.-Creación de grupos flexibles en inglés para los cursos de $1^{\circ}$ y $2^{\circ}$ de ESO.

4.-Creación de una asignatura de libre de disposición "conversaciones en lengua extranjera", para alumnos/as con problemas de aprendizaje en $1^{\circ}$ y de refuerzo positivo en $2^{\circ}$ (alumnado que tiene buenas aptitudes para los idiomas).

5.-Creación de las optativas de refuerzo de lengua y matemáticas en $1^{\circ}$ y $2^{\circ}$ de ESO.

6.-Creación de la asignatura de libre disposición "estrategias matemáticas" y mantenimiento de la asignatura "fomento a la lectura".

Se tomaron las medias de carácter individual siguientes:

1.- Programas de refuerzo de las materias pendientes de otros cursos (se designó la figura de un tutor de pendientes para la E.S.O.)

2.- Planes específicos para el alumnado que no promocionó.

3.- Programa de adaptación curricular y apoyos.

\section{6.- Metodología.}

$1^{\circ}$.- Reunión informativa con las familias del alumnado de primero y segundo de ESO para explicar cómo matricularse de forma correcta atendiendo a la nueva normativa. Explicación de todas las posibilidades, optativas, itinerarios, ofrecido por nuestro centro. 


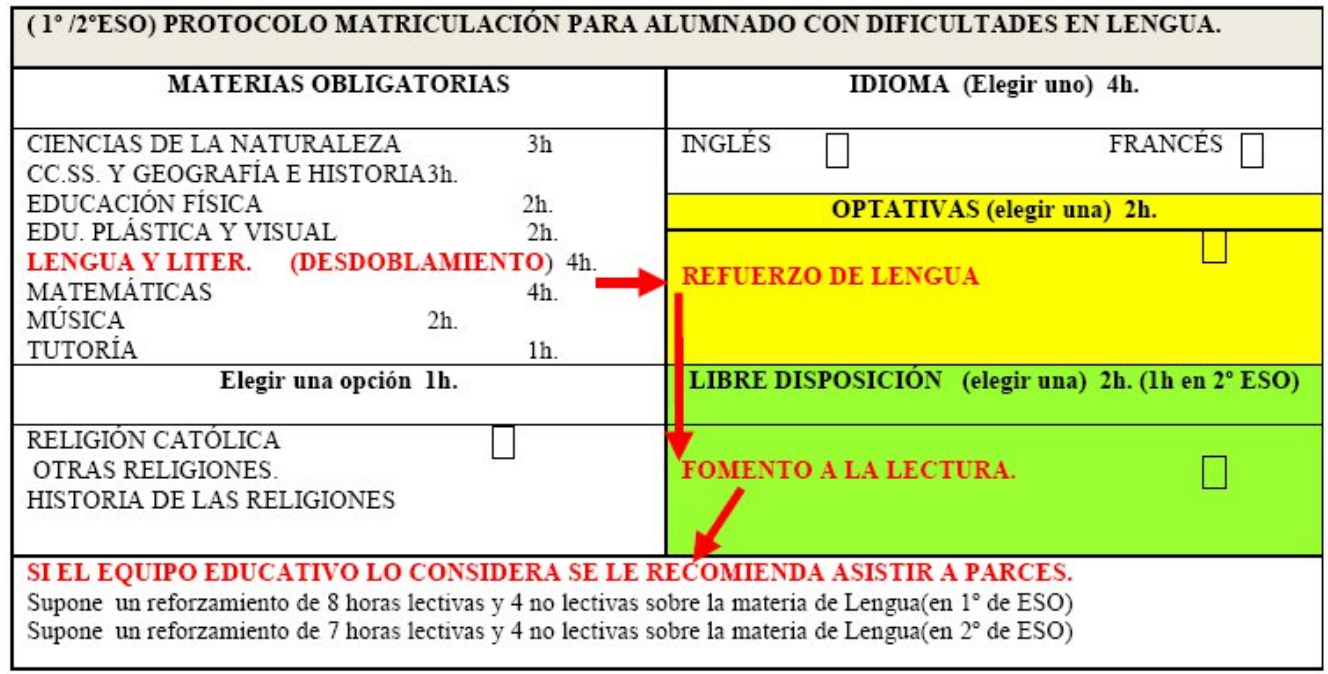

Figura 9.- Protocolo de matriculación para alumnado con dificultades en Lengua Castellana.

$2^{\circ}$.- Estudio del informe de tránsito para cotejarlo con las matrículas realizadas.

$3^{\circ}$.- Establecimiento de protocolos de matriculación.

\section{7.- Protocolos de matriculación:}

Los protocolos fueron cambiados, ya que una de las dificultades encontradas en el curso anterior fue la de reforzar correctamente al alumnado que presentaba dificultades en todas las materias instrumentales. Así, los nuevos protocolos implantados daban respuesta a todas las necesidades e incidían mucho más sobre las lagunas detectadas (mejoraban las actuaciones sobre las lenguas extranjeras y las matemáticas).

\section{A.-Alumnado con dificultades en lengua castellana (figura 9):}

$1^{\circ}$ Matriculado en grupo de desdoblamiento. $2^{\circ}$ Matriculado en refuerzo de lengua.

$3^{\circ}$ Matriculado en Fomento a la Lectura.

$4^{\circ}$ Opcionalmente y si era propuesto por el equipo educativo, se incluía en el programa P.A.R.C.E.S. (programa de apoyo y refuerzo educativo para secundaria).

B.-Alumnado con dificultades en matemáticas (figura 10):

$1^{\circ}$ Matriculado en grupo de desdoblamiento.

$2^{\circ}$ Matriculado en refuerzo de matemáticas.

$3^{\circ}$ Matriculado en estrategias matemáticas.

$4^{\circ}$ Opcionalmente y si era propuesto por el equipo educativo, se incluía en el programa P.A.R.C.E.S. (programa de apoyo y refuerzo educativo para secundaria).

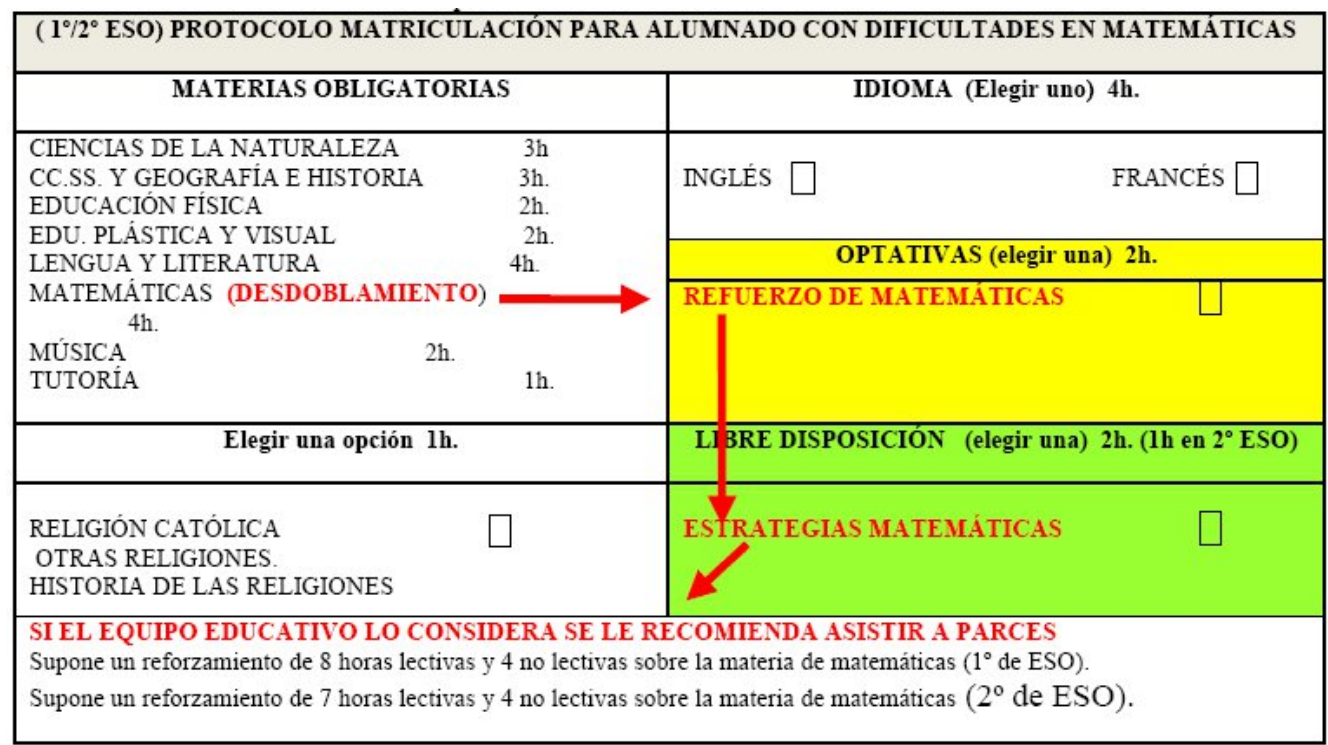

Figura 10.- Protocolo de matriculación para alumnado con dificultades en Matemáticas. 
Medidas educativas y organizativas de atención a la diversidad que mejoran el rendimiento escolar en el IES Sierra de los Filabres de Serón (Almería)

\begin{tabular}{|c|c|}
\hline \multicolumn{2}{|c|}{$\begin{array}{l}\text { (1\%/2 }{ }^{\circ} \text { ESO) PROTOCOLO MATRICULACIÓN PARA ALUMNADO CON DIFICULTADES EN MATEMÁTICAS } \\
\text { Y LENGUA CASTELLANA. }\end{array}$} \\
\hline MATERIAS OBLIGATORIAS & IDIOMA (Elegir uno) $4 \mathrm{~h}$. \\
\hline \multirow{4}{*}{ 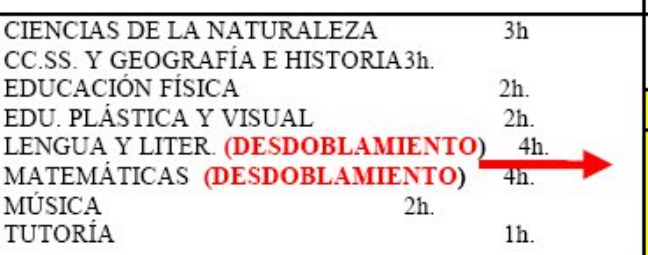 } & $\begin{array}{ll}\text { INGLÉS } & \text { FRANCÉS } \square\end{array}$ \\
\hline & OPTATIVAS (elegir una) $2 \mathrm{~h}$. \\
\hline & REFUERZO DE MATEMÁTICAS \\
\hline & \\
\hline Elegir una opción lh. & I IBRE DISPOSICIÓN (elegir una) $2 \mathrm{~h}$. (lh en $2^{\circ}$ ESO) \\
\hline $\begin{array}{l}\text { RELIGIÓN CATÓLICA } \\
\text { OTRAS RELIGIONES. } \\
\text { HISTORIA DE LAS RELIGIONES }\end{array}$ & FOMENTO A LA LECTURA. \\
\hline \multicolumn{2}{|c|}{$\begin{array}{l}\text { SI EL EQUIPO EDUCATIVO LO CONSIDERA SE LE RECOMIENDA ASISTIR A PARCES } \\
\text { Supone un reforzamiento de } 8 \text { horas lectivas y } 4 \text { no lectivas sobre la materia de lengua y matemáticas ( }\left(1^{\circ} \text { de ESO). }\right. \\
\text { Supone un reforzamiento de } 7 \text { horas lectivas y } 4 \text { no lectivas sobre la materia de lengua y matemáticas ( } 2^{\circ} \text { de ESO). }\end{array}$} \\
\hline
\end{tabular}

Figura 11.- Protocolo de matriculación para alumnado con dificultades en Matemáticas y en Lengua Castellana.

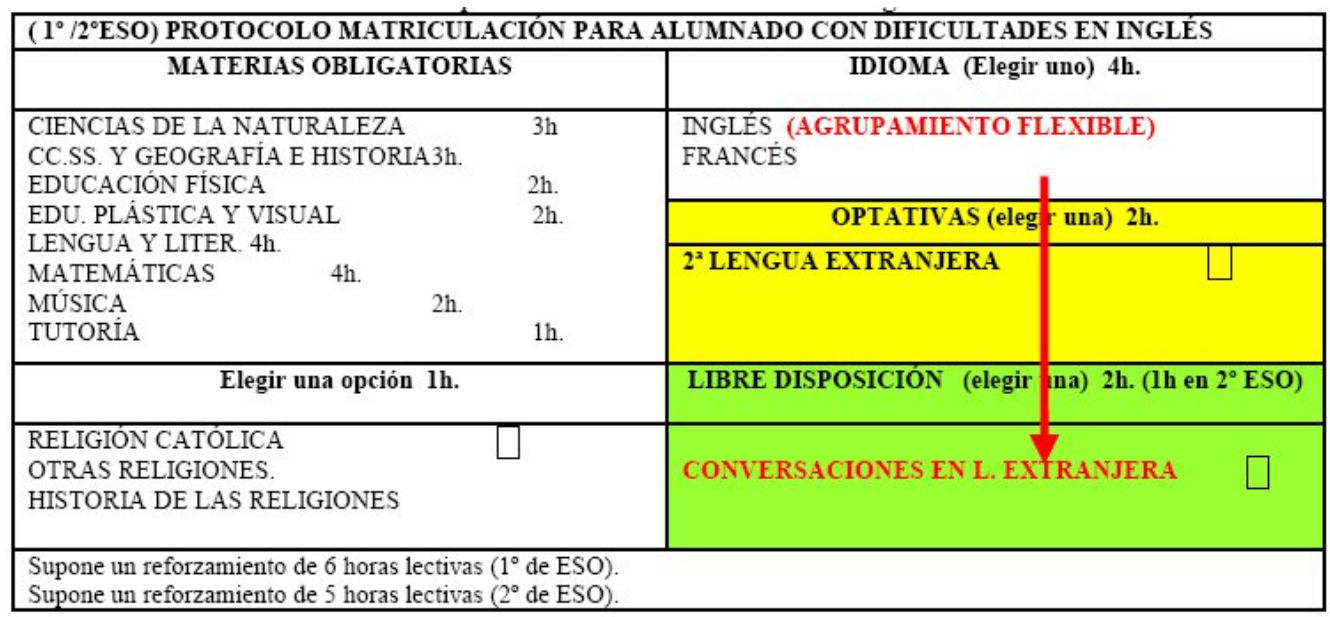

Figura 12.- Protocolo de matriculación para alumnado con dificultades en Inglés.

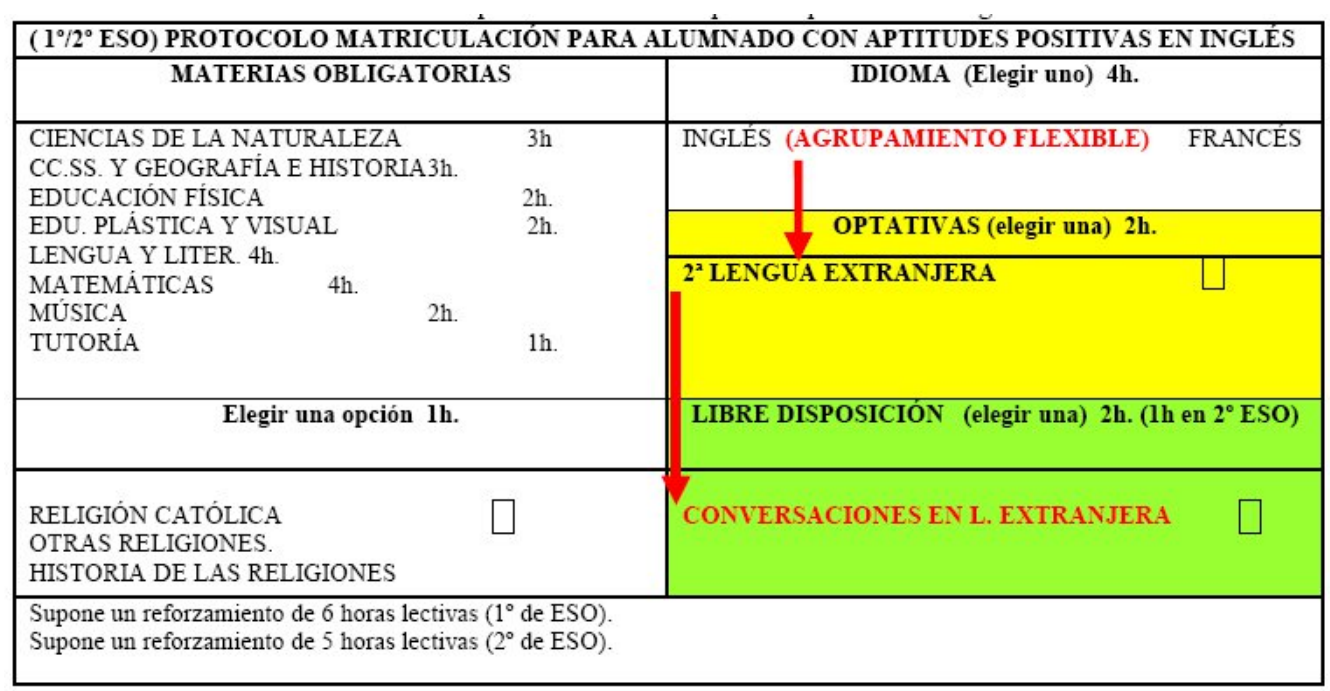

Figura 13.- Protocolo de matriculación para alumnado con aptitudes positivas en Inglés. 
C.-Alumnado con dificultades en lengua castellana y matemáticas (figura 11):

$1^{\circ}$ Matriculado en grupo de desdoblamiento de lengua y matemáticas.

$2^{\circ}$ Matriculado en refuerzo de matemáticas.

Tabla 4.- Comparativa de los resultados en lengua castellana para los tres cursos escolares.

\begin{tabular}{cccc}
\hline & $\begin{array}{c}\text { Suspensos } \\
\text { en Lengua } \\
(\mathbf{2 0 0 7 - 2 8})\end{array}$ & $\begin{array}{c}\text { Suspensos } \\
\text { en Lengua } \\
(\mathbf{2 0 0 8 - 0 9 )}\end{array}$ & $\begin{array}{c}\text { Suspensos } \\
\text { en Lengua } \\
(\mathbf{2 0 0 9 - 1 0 )}\end{array}$ \\
\hline $\mathbf{1}^{\mathbf{0}} \mathbf{E S O}$ & $31.6 \%$ & $17.8 \%$ & $22 \%$ \\
$\mathbf{2}^{\mathbf{0}} \mathrm{ESO}$ & $46.6 \%$ & $29.6 \%$ & $20 \%$ \\
TOTAL & $38.6 \%$ & $23.7 \%$ & $21 \%$ \\
\hline
\end{tabular}

$3^{\circ}$ Matriculado en Fomento a la Lectura.

$4^{\circ}$ Opcionalmente y si era propuesto por el equipo educativo, se incluía en el programa P.A.R.C.E.S. (programa de apoyo y refuerzo educativo para secundaria).

D.-Alumnado con dificultades en inglés (figura 12):

$1^{\mathrm{o}}$ Matriculado en agrupamiento flexible. escolares y un $9 \%$ mejor que el de la media andaluza (75.72\%). Se consiguieron mejorar los

Tabla 5.- Comparativa de los resultados en lengua castellana para los tres cursos escolares.

\begin{tabular}{cccc}
\hline & $\begin{array}{c}\text { Suspensos } \\
\text { Matemáticas } \\
(\mathbf{2 0 0 7 - 0 8 )}\end{array}$ & $\begin{array}{c}\text { Suspensos } \\
\text { Matemáticas } \\
(\mathbf{2 0 0 8 - 0 9 )}\end{array}$ & $\begin{array}{c}\text { Suspensos } \\
\text { Matemáticas } \\
(\mathbf{2 0 0 9 - 1 0 )}\end{array}$ \\
\hline $\mathbf{1}^{\mathbf{0}}$ ESO & $37.5 \%$ & $28.5 \%$ & $27.0 \%$ \\
$\mathbf{2}^{\mathbf{0}}$ ESO & $40.0 \%$ & $51.8 \%$ & $34.0 \%$ \\
TOTAL & $38.7 \%$ & $40.15 \%$ & $30.5 \%$ \\
\hline
\end{tabular}

Tabla 6.- Comparativa de los resultados en inglés para los tres cursos escolares.

\begin{tabular}{cccc}
\hline & $\begin{array}{c}\text { Suspensos } \\
\text { en Inglés } \\
(\mathbf{2 0 0 7 - 0 8 )}\end{array}$ & $\begin{array}{c}\text { Suspensos } \\
\text { en Inglés } \\
(\mathbf{2 0 0 8 - 0 9 )}\end{array}$ & $\begin{array}{c}\text { Suspensos } \\
\text { en Inglés } \\
\mathbf{( 2 0 0 9 - 1 0 )}\end{array}$ \\
\hline $\mathbf{1}^{\mathbf{0}} \mathbf{E S O}$ & $38.1 \%$ & $25.0 \%$ & $13 \%$ \\
\hline $\mathbf{2}^{\mathbf{0}}$ ESO & $40.0 \%$ & $51.8 \%$ & $17 \%$ \\
\hline TOTAL & $34.05 \%$ & $38.4 \%$ & $15 \%$ \\
\hline
\end{tabular}

resultados obtenidos en $1^{\circ}$ de ESO $(16.75 \%$ entre los dos cursos escolares), $2^{\circ}$ de ESO (4\% entre los dos cursos escolares), de $3^{\circ}$ de ESO (18.3\% en dos cursos escolares) y se mantuvieron los

\begin{tabular}{|c|c|c|c|c|}
\hline & $\begin{array}{l}\text { SERÓN } \\
\text { 2007-2008 }\end{array}$ & $\begin{array}{l}\text { SERÓN } \\
\text { 2008-2009 }\end{array}$ & $\begin{array}{l}\text { SERÓN } \\
\text { 2009-20010 }\end{array}$ & $\begin{array}{l}\text { ANDALUCÍA(Junta } \\
\text { de Andalucia, 2009) }\end{array}$ \\
\hline $1^{\circ} \mathrm{ESO}$ & $56.25 \%$ & $74 \%$ & $73 \% \quad \Rightarrow$ & $78.5 \%$ \\
\hline $2^{\circ} \mathrm{ESO}$ & $76.2 \%$ & $57 \%$ & $80 \%$ & $75.6 \%$ \\
\hline $3^{\circ} \mathrm{ESO}$ & $68 \%$ & $89 \%$ & $86.3 \%$ & $72 . \%$ \\
\hline $4^{\circ}$ ESO & $90.9 \%$ & $100 \%$ & $100 \%$ & $76.8 \%$ \\
\hline TOTALES & $72.83 \%$ & $80 \%$ & $84.8 \%$ & $75.72 \%$ \\
\hline
\end{tabular}

Figura 14.- Comparativa resultados alumnado promocionado/Titulado en los cursos 2007-2008, 2008-2009 y 2009-2010.

$2^{\circ}$ Matriculado en conversaciones en lengua extranjera.

E.-Alumnado con aptitudes positivas en inglés (figura 13):

$1^{\circ}$ Matriculado en agrupamiento flexible (en positivo)

$2^{\circ}$ Matriculado en segunda lengua extranjera.

$3^{\circ}$ Matriculado en conversaciones en lengua extranjera.

\subsection{Conclusiones al término del curso escolar 2009-2010.}

Se consiguió el objetivo de elevar la tasa total de alumnado que promocionaba o titulaba $(72.83 \%$ para el curso 2007-2008) elevándolo hasta el $84.8 \%$, un $11.9 \%$ de mejora en dos cursos excelentes resultados en $4^{\circ}$ de la ESO (siempre entorno al $100 \%$ de alumnado titulado) (figura 14).

Se consiguió elevar el éxito en la asignatura de lengua castellana (tabla 4) y específicamente en segundo curso donde el fracaso se situaba en el $46.6 \%$. En $1^{\circ}$ de ESO se redujo, en el periodo de dos cursos escolares, en un $9.25 \%$. En $2^{\circ}$ de ESO en $26.6 \%$. Arrojando unos datos generales de mejora, para el alumnado del primer ciclo de secundaria, de un $17.6 \%$.

Se consiguió elevar el éxito en la asignatura de matemáticas en ambos cursos $\left(1^{\circ}\right.$ y $2^{\circ}$ de ESO). En el primero de ellos con una mejora de un $10.5 \%$, mientras para el segundo, dicha mejoría alcanzó el 6\%. Estos datos arrojaron una mejoría global del 8.2\% (tabla 5). 
Se consiguió también elevar el éxito en la asignatura de inglés (tabla 6). Tras no haber aplicado ninguna herramienta educativa relacionada con la atención a la diversidad durante el primer curso escolar, la planificación de las actuaciones durante el curso 2009-2010 dieron el resultado esperado.

Disminuyeron los alumnos/as con fracaso en esta asignatura tanto en $1^{\circ}$ como en $2^{\circ}$ de ESO. En el primer caso en un $25.1 \%$ y en el segundo en un $23 \%$. Con una disminución global del fracaso escolar en dicha asignatura del 19.05\%.

En cuanto a la evaluación de diagnóstico se refiere (Junta de Andalucía, 2010), (figura 15), los resultados en lengua castellana se mantuvieron por encima de los 550 puntos transformados mientras en matemáticas siguieron estando en la media de los 500 puntos. primeros cursos de Enseñanza Secundaria Obligatoria. Los claustros y equipos directivos de los centros educativos andaluces, tienen en sus manos una extraordinaria herramienta, que a la luz de este trabajo, se muestra como muy eficaz para la consecución de la mejora de los resultados académicos de los alumnos y alumnas a través del adecuado tratamiento a la diversidad, que en definitiva es lo que nos propone esta ley.

Los índices de promoción y titulación fueron elevados sustancialmente así como se consiguió bajar las tasas de fracaso escolar en las asignaturas instrumentales. La no modificación de los resultados obtenidos en las pruebas de diagnóstico puede deberse más a factores externos que a falta de capacidad y competencia (aspectos relevantes medidos por estas pruebas). Problemas familiares, inadaptación al entorno, ritmos de aprendizaje diferentes, falta de atención individualizada, problemas específicos con el estudio de Tabla 7. Comparativa para el curso escolar 2009-2010

\begin{tabular}{cccc}
\hline & $\begin{array}{c}\text { COMUNICACIÓN } \\
\text { LINGǗSTICA }\end{array}$ & $\begin{array}{c}\text { COMUNICACIÓN } \\
\text { MATEMÁTICA }\end{array}$ & $\begin{array}{c}\text { MUNDO } \\
\text { FíSICO }\end{array}$ \\
\hline ESPAÑA & 500 & 500 & 500 \\
ANDALUCÍA & 498 & 488 & 500 \\
SERÓN & 557.36 & 501.07 & 454.97 \\
\hline
\end{tabular}
algunas asignaturas, ratios muy altas, etc., etc., constituyen el obstáculo principal para el éxito escolar y por tanto, con medidas como las realizadas en el IES Sierra de los Filabres, deben y pueden

No parece que hubiera grandes diferencias en los resultados de dichas pruebas tras la aplicación de las medidas de atención a la diversidad. No obstante se desprenden unos resultados medios, ligeramente superiores a los obtenidos (como media), por los escolares andaluces y españoles (Ministerio de Educación, 2010) (tabla 7).

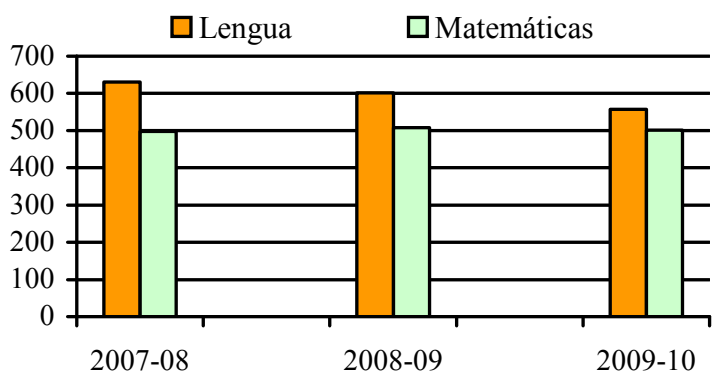

Figura 15.- Distribución del alumnado en los desdoblamientos.

\section{4.- CONCLUSIONES FINALES.}

Queda demostrado que la adopción de ciertas medidas de carácter organizativo, bien planificadas, pueden influir muy positivamente en el rendimiento del alumnado que estudia los contribuir a la mejora de los rendimientos escolares.

\section{5.- REFERENCIAS BIBLIOGRÁFICAS.}

Álvarez, V., Rodríguez, A., García, E., Gil, J., Romero, S., Padilla, M ${ }^{a}$ Teresa., et al. (2002). La atención a la diversidad en los centros de enseñanza secundaria: estudio descriptivo en la provincia de Sevilla. Revista de Investigación Educativa. Volumen 20, (nº 1), páginas 225-245.

Arnaiz, P. \& Haro, R. de (1997). Educación intercultural y atención a la diversidad. En F. Salinas y E. Moreno (Coord). Semejanzas, Diferencias e Intervención Educativa. Granada: Fundación, Educación y Futuro.

Consejería de Educación (2010). Evaluación de diagnóstico. (2009-2010). Sevilla: Junta de Andalucía.

Consejo Escolar del Estado. (2010). Informe sobre el Estado y Situación del Sistema Educativo. Madrid: Secretaría general Técnica. Ministerio de Educación.

Departamento de Orientación y Equipo Directivo. (2010). Medidas educativas y organizativas que mejoran el rendimiento escolar y disminuyen el abandono. Datos que hablan de calidad. Monográficos Escuela. (Febrero de 2010), páginas 20, 21 y 22. 
Dirección General de Ordenación y Evaluación Educativa. (2009). El Sistema Educativo en Andalucía. Indicadores del Curso 2007-2008. Sevilla: Junta de Andalucía.
Dirección General de Participación e Innovación Educativa. (2010). Observatorio para la convivencia escolar en Andalucía. Informe anual 2009-2010. Sevilla: Junta de Andalucía. 